\title{
Capnocytophaga canimorsus sepsis: two clinical cases at the Bolzano Regional Hospital
}

\author{
Patrizia Innocenti', Richard Aschbacher', Brigitte Ladinser', Renate Meyer', Ludwig Moroder', Elisabetta Pagani', \\ Ulrich Josef BaumgartI' ${ }^{2}$, Bernadette Moser $^{3}$, Clara Larcher' \\ I Laboratorio Aziendale di Microbiologia e Virologia, Comprensorio Sanitario di Bolzano, Bolzano \\ 2 Pneumologia, Comprensorio Sanitario di Bolzano \\ 3 Anestesia e Rianimazione, Comprensorio Sanitario di Bolzano, Bolzano
}

Key words: Capnocytophaga canimorsus, Sepsis, Postsplenectomy infections, Zoonosis

Sepsi da Capnocytophaga canimorsus: due casi clinici nell'ospedale di Bolzano

\section{SUMMARY}

Capnocytophaga canimorsus is a commensal bacterium in the oral cavity of dogs and cats and has been isolated from humans with a history of dog or cat bites or scratches. C. canimorsus are capnophilic facultative anaerobic, fastidious gram negative fusiform rods, included in the CDC group DF-2 or dysgonic fermenter 2. The bacterium can cause severe illness in patients at elevated risk i.e. after splenectomy, with alcohol addiction, chronic lung or liver diseases or immunosuppression. In 2010 at the Bolzano Regional Hospital (Northern-Italy) C. canimorsus was isolated from blood cultures in two splenectomised patients suffering from septicaemia. The bacterium is difficult to cultivate because of its specific requirements for nutrients as well as to identify with routine automated instruments; therefore we used molecular typing by I6S rDNA sequencing. The true number of $C$. canimorsus infections is probably underestimated due to the fastidious growth of the organism. However, given the severity of the disease and the poor outcome one should consider this bacterium to apply appropriate diagnostic methods for this etiologic agent.

\section{INTRODUZIONE}

Capnocytophaga canimorsus è un bacillo Gram negativo, capnofilico, compreso nel gruppo CDC Dysgonic fermenters 2; a differenza di altre specie di Capnocytophaga (es. C. gingivalis, C. sputigena), che colonizzano anche le mucose orali e gengivali dell'uomo, $C$. canimorsus si ritrova nelle mucose orali di cane e gatto ed è occasionale agente d'infezioni zoonotiche nell'uomo, soprattutto in soggetti immunodepressi, alcolisti, con malattie respiratorie croniche ed asplenici.

Le manifestazioni cliniche possono essere talora gravi come la sepsi, la meningite e sono conseguenti a morsi d'animali o contatto con le loro secrezioni salivari $(1,2,3)$.

La prima descrizione di questo agente patogeno risale al 1976 e da allora un numero relativamente piccolo di casi è stato documentato, probabilmente a causa delle difficoltà a coltivare ed identificare correttamente questo microrganismo (3). Il batterio, infatti, cresce lentamente sui normali terreni al sangue (Columbia Agar al 5\% sangue di montone), non cresce su Agar MacConkey, abbisognerebbe di concentrazioni elevate di ferro; inoltre i metodi automatizzati utilizzati di routine per l'identificazione non sono in grado di riconscerlo.

Nei primi mesi del 2010 nell'Ospedale di Bolzano è stata isolata dal sangue di due pazienti con segni di sepsi grave.

\section{CASI CLINICI}

Il primo paziente, di 42 anni, splenectomizzato, abituale consumatore di alcolici e possessore di cani, è giunto al pronto soccorso in shock settico e presentava sindrome da disfunzione multiorgano e petecchie. La terapia empirica è stata eseguita con levofloxacina e ceftriaxone, ed è continuata anche dopo l'identificazione del microrganismo.

Il paziente è sopravvissuto, ma necrosi estese ne hanno richiesto l'amputazione delle gambe e di alcune falangi delle mani.

La seconda paziente di 75 anni era anch'essa splenectomizzata, diabetica, portatrice di tracheostoma con storia pregressa di carcinoma alla laringe; ricoverata in pneumologia mostrava segni di sepsi, polmonite e grave scompenso cardiaco ed è deceduta nonostante la terapia empirica con ceftriaxone e azitromicina.

\section{MATERIALI E METODI}

Sono stati utilizzati i flaconi per emocoltura (FA, FN Bact/Alert, bioMérieux), incubati per 5 giorni e le subcolture sono state allestite in $\mathrm{CO}_{2}$ al $5 \%$ su Agar sangue (COS bioMérieux) ed Agar cioccolato (PVX bioMérieux), Schaedeler Agar (SCS bioMérieux) in anaerobiosi se si trattava di flaconi anaerobi. Il sistema di ruotine utilizzato per l'identificazione ed antibiogramma è Vitek2 (bioMérieux). Al fine d'identificare il microrganismo isolato, il sequenziamento genico della regione $16 \mathrm{SrDNA}$ è stato condotto utilizzando lo strumento ABI Prism 3130 Genetic Analyzer (Applied Biosystems), secondo metodo di Sanger, previa estrazione degli acidi nucleici con il sistema automatizzato NucliSENS $^{\circledR}$ easyMAG ${ }^{\mathrm{TM}}$ (bioMérieux Italia) da una sospensione colturale, ed ottenimento dei relativi ampliconi per adattamento dei protocolli descritti da Chen C, et al.(4).

Le sequenze ottenute sono state allineate utilizzando il database blast/GenBank (http://www.ncbi.nlm.nih.gov/blast/Blast.cgi).

\section{RISULTATI}

La positivizzazione dei flaconi per emocoltura è avvenuta in 2-4 giorni; per un paziente si è evidenziata positività in 3 flaconi su 4 (due anaerobi ed un aerobio) e in due su due per il secondo paziente (flacone anaerobio + aerobio).

All'esame microscopico diretto si notavano bacilletti fusiformi Gram negativi (Figura I).

La crescita su Agar sangue ed agar cioccolato ha permesso di evidenziare in 3-4 giorni colonie piccole, trasparenti, ossidasi e catalasi positive.

Il sequenziamento genico, a cui si è ricorsi dopo il fallimento dell'identificazione mediante i sistemi automatici utilizzati di routine (Vitek2), ha permesso d'identificare in entrambi i casi il microrganismo come Capnocytophaga canimorsus, con un omologia del $99 \%$ nei confronti del ceppi identificati con accession number FJ669151 e FJ669153.

\section{CONCLUSIONI}

C. canimorsus è difficile da coltivare e da identificare con sistemi automatici tradizionali; tuttavia il suo ritrovamento quale agente causale di sepsi nei casi descritti dimostra la

\section{Corresponding author: Patrizia Innocenti}

Laboratorio Aziendale di Microbiologia e Virologia; Comprensorio Sanitario di Bolzano

Via Amba Alagi, 5 - 39100 Bolzano - Tel.: 047। 909627 - Fax: 047। 27263।

E-mail: patrizia.innocenti@asbz.it 


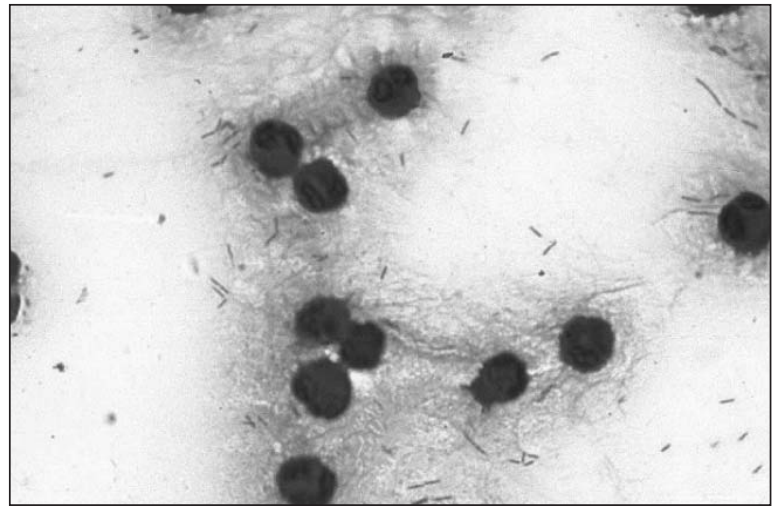

Figura I. Capnocytophaga canimorsus da emocoltura.

probabile sottostima delle infezioni causate da C. canimorsus, ribadendo l'importanza del sequenziamento genico nell'identificazione di microrganismi "fastidious" isolati da emocolture.
La gestione di pazienti critici con particolari fattori di rischio (splenectomia, pazienti immunodepressi, alcolisti) deve tenere presente la possibilità d'isolamento di tali germi in attesa di conferme da parte del Laboratorio di Microbiologia.

Per la terapia la prima scelta cade sulla penicillina $G$, tuttavia C. canimorsus è descritta essere sensibile anche ad imipenem, clindamicina, cefalosporine di terza generazione e fluorochinoloni (3) e pertanto di regola non presenta particolari problemi terapeutici, anche in caso di terapia empirica necessaria per la dilazione dei tempi di refertazione.

\section{BIBLIOGRAFIA}

1. Ohler RL, Velez AP, Mizrachi M, Lamarche J, Gompf S. Bite related and septic syndromes caused by cats and dogs. Lancet Infect Dis 2009; 9 (7): $439-47$

2. Janda JM, Graves MH, Lindquist D, Probert WS. Diagnosing Capnocytophaga canimorus Infections. Emerging Infectious Disease, 2006; 12 (2).

3. Gaastra Wim, Lipman Len JA. Capnocytophaga canimorus (Rewiew). Veter Microb 2010; 140: 339-46.

4. Chen C, et al. J Clin Microbiol 2000; J Clin Microbiol, 2001. 